\title{
Are Eating Disorders Related to Attention Deficit/Hyperactivity Disorder?
}

\section{Shauna P. Reinblatt, MD}

\section{Address}

Department of Psychiatry and Behavioral Sciences, Division of Child Psychiatry, Johns Hopkins University School of Medicine, 550 North Broadway, Room 206 D, Baltimore, MD, 21205, USA

Email: Shauna.reinblatt@jhmi.edu

Published online: 9 0ctober 2015

(C) Springer International Publishing AG 2015

This article is part of the Topical Collection on Eating and Other Impulse

Control Disorders

Keywords Binge eating - Loss of control eating - Eating disorder - Attention deficit/hyperactivity disorder (ADHD) · Bulimia nervosa

\section{Opinion statement}

Attention deficit/hyperactivity disorder (ADHD) is a disorder characterized by impulsivity, hyperactivity, and inattention. Binge-eating behavior is often impulsive and is the hallmark of the two eating disorders, binge-eating disorder (BED) and bulimia nervosa (BN), both of which are associated with significant health impairment. Bingeing behavior is also seen in the binge purge subtype of anorexia nervosa. Individuals with $\mathrm{AN}$ of the binge purge subtypes, BN and BED, have been found to exhibit impulsive behaviors that are often not limited to binge eating alone. There is preliminary evidence linking ADHD to $\mathrm{BN}$ and to BED in both adults and children. The neurobiological mechanisms behind these associations are only beginning to emerge; however, they suggest that impulse control deficits may play a role in these eating disorders. Additionally, although they may not meet full criteria for one of these eating disorders, some adults and children with ADHD present with dysregulated, impulsive eating disorder behaviors and there is a growing association between ADHD, obesity, and binge-eating behavior in both children and adults. The relationship between ADHD and binge eating is novel, supported by growing evidence and worthy of further research. We will review the underlying neurobiological underpinnings, neuroimaging data, and possible psychopharmacological treatment options, which target both ADHD and binge-eating behaviors as well as future research and treatment directions. 


\section{Introduction}

Attention deficit/hyperactivity disorder (ADHD) is defined in Diagnostic and Statistical Manual-5 (DSM-5) by symptoms of persistent inattention and/or hyperactivity and impulsivity in two or more settings, beginning prior to 12 years of age. ADHD has two subtypes, inattentive and hyperactive/impulsive, and is a common impairing disorder often accompanied by comorbid psychiatric conditions such as anxiety or depression. It has been estimated to occur in $5 \%$ of children in the United States by the DSM-5 $[1,2]$. Eating disorders, including binge purge-type anorexia nervosa (AN-P), bulimia nervosa (BN), and binge-eating disorder (BED), also frequently co-occur with anxiety, mood, or substance abuse disorders and result in poor function and physical complications with significant public health implications. Both ADHD and eating disorders tend to begin early in life but can be chronic and persist into adulthood. We will focus predominantly on BN and BED since most of the evidence discussing possible links between eating disorders and ADHD have focused on these two disorders, both of which are more common than AN-P. This manuscript will present adult data except where the term child or adolescent is used as data are available.

Officially introduced in DSM-5, BED is a relatively new diagnosis and is the most common of the feeding and eating disorders with an estimated lifetime prevalence of $3.5 \%$ in adults [3]. Binge eating in $\mathrm{BN}$ and BED is recurrent and occurs at least once a week for 3 months or more, with binge-eating episodes defined as (1) eating more than most people would eat within a discrete (2 h) period of time and (2) a sense of loss of control over eating. In addition to these binge-eating episodes, the diagnosis of $\mathrm{BN}$ also requires compensatory behaviors to prevent weight gain (such as purging, laxatives, or exercise) and an undue influence of body shape or weight on self-image. BED lacks the compensatory behaviors seen in $\mathrm{BN}$, and binge eating in BED must feature at least three associated symptoms (such as eating alone; eating rapidly during a binge; eating in the absence of hunger; and eating until uncomfortably full or feelings of shame, embarrassment, or guilt associated with binge behavior). In children, the term loss of control (LOC) eating is often used to capture disinhibited eating symptoms and a wider phenotype of BED, which does not require an objectively large amount of food yet still requires loss of control and associated emotional and behavioral symptoms. Below, we will discuss possible links between ADHD and eating disorders focusing first on $\mathrm{BN}$ and $\mathrm{AN}-\mathrm{P}$ and later on the more recently defined diagnosis of BED as well as implications for treatment with a focus on the most relevant recent studies.

\section{Relationship between ADHD and eating disorders (AN-P and BN)}

ADHD symptoms and dysregulated eating behaviors have been frequently associated in the extant literature. The prevalence of eating disorders in ADHD samples has been reported across a range up to $12 \%$ [4-6]. Conversely, the prevalence of ADHD symptoms has been reported to be from 5 to $17 \%$ in samples of eating disorder patients $[7,8]$. A 5-year prospective study found that children with ADHD-combined type demonstrated more BN-type eating pathology at follow-up than those without ADHD [9], with impulsivity being the strongest predictor of eating disorder behavior examined. In a second study, $9 \%$ of bulimic inpatients exhibited comorbid [10] ADHD suggesting an elevated co-occurrence of ADHD in this eating disorder. Finally, elevated childhood histories of ADHD have been reported in adult clinical samples with $\mathrm{BN}$ and AN-P [8].

The risk of developing $\mathrm{BN}$ has been associated with impulsivity and inattention [11]. In one study, girls with ADHD were 3.6 times more likely to have any eating disorder than girls without ADHD and 5.6 times more likely to specifically present with $\mathrm{BN}$ [12]. A prospective follow-up of the Multimodal 
Treatment Study of Children with ADHD found that children (boys and girls) had an increased risk for BN symptoms (compared with a control group) and that childhood impulsivity predicted BN symptom onset in adolescence 8 years later [13]. The recent secondary data analysis of the National Longitudinal Study of Adolescent Health found that although those with clinical ADHD are more likely to experience eating disorder symptoms (binge/purge and restrictive), those with subclinical ADHD were only more likely to present bingeing

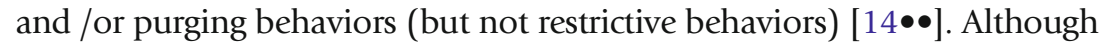
another study that utilized self-report measures reported negative findings of an association between BN and ADHD in adult women, its small sample size $(n=32)$ suggests that it was underpowered to demonstrate a link between ADHD and BN [15].

Taken together, these data suggest that among individuals with eating disorder symptomatology, there is a greater risk of ADHD in those with binge-eating/purging behaviors (such as in $\mathrm{BN}$ or AN-P) rather than restricting behaviors alone. Additional studies examining the comparative rates of ADHD phenomenology in AN of the purging subtype versus restricting subtype would lend support to this hypothesis and may be helpful in clarifying underlying mechanisms.

\section{Relationship between ADHD and obesity}

Adults and children with ADHD have been reported in both epidemiologic and clinical studies to have a significantly greater risk of also presenting with obesity $[16,17]$. Conversely, those who are overweight/obese also have been found to have a greater likelihood of presenting with ADHD. In treatment seeking obese adults, one study found that $25 \%$ met criteria for ADHD, with a higher prevalence in the most obese [18]. In overweight youth, studies have found up to $50 \%$ of youths treated for overweight had ADHD [19] while $20 \%$ of youths treated for ADHD were overweight [20]. A large epidemiologic study replicated these findings and reported that those not treated with stimulants were 1.5 times more likely to be overweight, whereas those treated with stimulants were 1.6 times more likely to be underweight (versus children without these diagnoses) [17]. The risk of obesity with ADHD also holds true longitudinally, as shown in a 33-year follow-up study of males with ADHD who were 2 times more likely to be obese at follow-up than controls [21]. Taken together, the evidence shows that overweight, BN, and binge-eating behavior all occur more than expected in people with ADHD [22-24]. We also have good evidence that BED and LOC eating are associated with increased weight status in both children and adults [25].

\section{Relationship between ADHD and binge-eating disorder}

Given associations between ADHD and obesity and obesity and binge behavior, the question arises whether ADHD and binge-eating disorder are related? Most early studies of the past decade that examined the relationship of bingeing and ADHD did not examine BED, probably in part because it was still a research diagnosis in DSM-4. One early study of BED however did find that it was frequently comorbid with ADHD [5] and ADHD has been reported to be linked 
to loss of control eating [26]. ADHD was also associated with binge eating and emotional eating in several non-clinical samples including a study of a community sample [22] and a large epidemiological study which reported an association between subclinical ADHD and binge-eating disorder behaviors [27]. Another epidemiologic study found that the relationship between ADHD and weight in adults may have been affected by binge eating. Impulsivity (as explored below) may be one hypothesized mechanism influencing the relationship between BED and ADHD. Although another study reported that ADHD symptoms were associated with binge eating and some measures of impulsivity, they did not find that impulsivity mediated the relationship between ADHD and BED [28].

Although the literature on BED in children is still scant, an association between binge-eating behavior and ADHD is also generally found in epidemiologic studies of children and adolescents. One large epidemiologic study reported that childhood ADHD symptoms increased the risk of binge eating

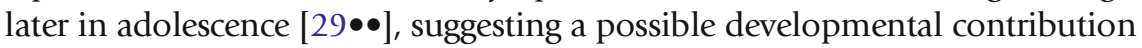
to eating behavior similar to maturational delays seen in ADHD. In a retrospective review performed by our group, 252 pediatric charts were reviewed in two community child mental health clinics [30]. Children with ADHD were found to be significantly more likely to be overweight/obese than children without ADHD. There was a statistically significant association between ADHD and binge eating; $26 \%$ of children with ADHD had binge-eating behaviors (versus only $2 \%$ of children without ADHD). However, it is noteworthy that $74 \%$ of children with ADHD did not binge eat. These findings suggest the possibility of a subgroup of children with ADHD who present with dysregulated binge eating and may represent an endophenotype that warrants further study. Further research should examine whether this subgroup of children with ADHD are more impulsive or have more extreme symptoms or comorbid mood and anxiety disorders when compared to those with ADHD but without binge eating. As cross-sectional data preclude causal conclusions, prospective data are required, particularly in children to establish whether ADHD predisposes to binge eating or whether an underlying personality trait such as impulsivity is a common risk factor for both conditions. Finally, neuroimaging studies may help clarify the underlying neurocircuitry affected in these individuals with the goal of developing more targeted treatments in the future.

\section{Proposed neuropsychological underpinnings of the ADHD/ED relationship}

Children with binge eating may present clinically with dysregulated eating patterns, and children with ADHD may present with generally dysregulated behaviors. For example, males with ADHD have been found to exhibit impulsive eating patterns (at irregular meal times) and generally ate foods of lesser nutritional status (fewer fruits and vegetables and more sweetened beverages) [31].

Executive function deficits (such as attentional problems and impulsive behavior) have emerged as common neurocognitive features to obesity, eating disorders, and ADHD. AN and BN participants have been reported to 
demonstrate deficits in attention on psychological testing [32]. Attentional biases to food describe the attention given to food stimuli. In individuals with an eating disorder, it is unclear however whether attentional biases to food stimuli are related to anxiety around eating (and a desire to restrict) or to craving for the food [33•]. In overweight/obese individuals, findings do not always support a hypothesis of attentional bias for higher-calorie food. Experiments where participants are distracted (by television or computer) influenced later food consumption, by impacting meal memory. Manipulating attention appears to affect later-eating behavior, perhaps appetite [34]. One requires sufficient capacity to attend to the task of eating. It is conceivable that symptomatic overlap of inattention found in ADHD may contribute to bulimic behaviors; bulimic patients may present with impulsive interruptions of their activities to binge eat, possibly due to associated attention problems [11].

Impulsivity may be characterized by the inability to inhibit a response or a deficit in inhibitory control [35]. Inhibitory control deficits have been welldescribed in the pediatric ADHD literature as a primary deficit [35]. Youths with ADHD and inhibitory control problems show deficits on response inhibition tasks, including Go/No-Go tasks. Besides response inhibition, impulsivity is impacted by reward sensitivity and food is a natural reward. The reward system uses motivation to determine behavior based on learning via the dopaminergic mesolimbic pathways [36], and dopaminergic reward pathways are implicated in ADHD [37]. Both the animal and human literature suggest that palatable food high in fat and sugar activates the dopaminergic reward system and results in eventual dysregulation of the homeostatic feeding drive as the consumption of palatable food becomes more driven and compelling in some individuals with binge-type behaviors despite increasing negative consequences. The term food addiction "has been used to describe the similarity in escalating consumption of palatable high-calorie-density foods in some individuals to drug addiction [38]. Unlike drug addiction, however, there is not a distinct food type or withdrawal syndrome that characterizes binge behavior, whereby food is very rewarding and acts like a drug of addiction; however, there is little data regarding withdrawal symptoms as in drugs of abuse. The ability to process the motivational value of stimuli or the reinforcing properties may be different in BED. Genetic studies support the idea that individuals with BED may have greater reward responsiveness based on the Taq1A polymorphism of the ANKK1 gene which is a genetic marker for dopamine [39•]; notably, D2 dopamine receptors in the striatum may affect reward signaling [40]. An individual's ability to inhibit responses could also modulate their ability to delay reward. Perhaps these interrelated neurobehavioral factors also apply when there is comorbid ADHD and binge eating.

Irrespective of $\mathrm{ADHD}$, obese individuals have been described as being more impulsive, with poorer impulse control than leaner individuals [41]. Overall, overweight individuals appear to be less able to inhibit responses, particularly for palatable food. Impulsivity has been posited as a contributing neurobiological factor for binge-eating behaviors; however, the data in adults are not clear. A study of young women with BED found deficits in impulse control but only in the context of emotional stressors and negative affect [42]. Another study involving obese adults (with or without BED) failed to find any differences in executive function [43]. 
There are data regarding the role of impulsivity in children with binge-eating behavior. Our group conducted a study of 79 children examining parental ratings of impulsivity (BRIEF executive function measure) as well as behavioral tasks (Go/No-Go task) [26]. We examined children with binge eating who fit putative criteria for symptoms of loss of control eating disorder (LOC-ES) [25]. Children with LOC-ES showed poorer response inhibition (or were more impulsive) than children who did not binge. Additionally, overweight and obese children (with LOC-ES) presented significantly greater odds of ADHD compared to overweight and obese children (without LOC-ES). Contrastingly, another pediatric study examining episodes of LOC eating failed to find differences in impulsivity in a slightly older sample [44]. Notably, post hoc analyses in Hartmann et al.'s study revealed that eight of the children had symptoms of both LOC and ADHD. The relationships between obesity, binge eating, impulsivity, and ADHD are complex and difficult to examine in crosssectional studies. These studies suggest that prospective studies examining the neurobiological underpinnings of binge eating and ADHD would enhance our knowledge of these disorders.

\section{Neuroimaging findings supporting the ADHD/binge eating relationship}

Although we do not know of any neuroimaging studies directly examining comorbid ADHD and BED, which would greatly enhance our understanding of the association, there are some studies examining binge eating. There are positron emission tomography (PET) studies examining adult binge eating. Karhunen's [45] PET study examined women with BED and found increased cerebral blood flow in the left frontal and prefrontal brain areas in response to food (compared to controls). Although the significance of these brain areas with regards to eating behaviors are not yet fully understood, they may have a role in the cognitive control of eating. Secondly, Wang et al. [46••] used PET to examine dopamine in women (comparing BED versus non-BED). The stimulant methylphenidate was given to enhance the dopamine signal. When food stimuli were given with methylphenidate, there were significant increases of dopamine in the caudate in the women with BED but not those without BED. Thus, the neurotransmitter dopamine in the caudate may be important to the underlying phenomenology of BED. It is noteworthy that methylphenidate is also used in the treatment of ADHD. Dopamine transmission in the caudate may be important factor in binge eating, just as it has been suggested in ADHD [47].

Relatively few functional magnetic resonance (fMRI) imaging studies have been done on binge eating or full criteria BED. A study by Balodis et al. [48] reported decreased recruitment of reward circuitry (involving frontostriatal areas) in individuals with persistent bingeing post-treatment. A recent study by Geliebter et al. [49] demonstrated heightened responsivity of the anterior cingulate cortex (ACC) in response to high-energy-food cues in adults with subthreshold BED. ACC and orbitofrontal cortex activation was correlated with reward sensitivity in another study of BED, lending support for different brain activation patterns than non-BED individuals [50]. 
Similar findings involving the ACC and the prefrontal cortex have also been reported in children. Lock et al. [51] reported increased activation in the ACC, precentral gyrus, and the dorsolateral prefrontal cortex during a Go/No-Go task in youth with AN-P and BN. Taken together, PET and fMRI studies in individuals with binge eating present support for involvement of areas of the brain implicated with impulse control and reward pathways. It is noteworthy that activation of the ACC has also been shown to play a role in ADHD [52]. ADHD symptoms have been found to be related to the right anterior attention network which impacts inhibitory control. Additionally, the reward system has also been implicated in ADHD [37].

Other fMRI studies performed in adults with BN (who binge eat) have found associations with brain pathways that influence self-regulation [53].

Frontostriatal circuitry dysfunction relating to deficits in self-regulation has been reported [54]. A study of adolescents with BN also reported frontostriatal system involvement which could also impact on self-control and impulsive behaviors.

Taken together, these neuroimaging findings tend to support involvement in the frontostriatal pathways in binge eating. More neuroimaging studies focusing specifically on full-syndrome BED and overlapping ADHD samples are needed to determine possible overlapping neurocircuitry of binge eating and ADHD and determine underlying neurobehavioral underpinnings of these disorders.

\section{ADHD-targeted pharmacological treatments that may be useful for BED}

Since the phenomenology of BED, like ADHD, includes impulsive behavior deficits, a medication that targets impulsive behavior in ADHD could hypothetically have value in pediatric BED. Indeed, there is some evidence that in adults, medications that are effective in ADHD may have some efficacy in binge eating, including stimulant medications and atomoxetine.

The stimulant medication lisdexamfetamine (LDX) recently became the first medication to be Food and Drug Administration (FDA) approved to treat mod-

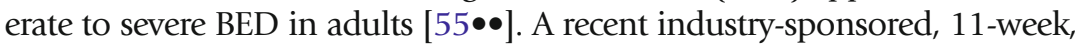
randomized controlled trial of LDX was shown to decrease the number of binge eating days per week when administered at doses of 50 to $70 \mathrm{mg} /$ day (but not $30 \mathrm{mg}$ ) in adults with moderate to severe BED. There were 259 participants; dosages of LDX were maintained for 8 weeks after a 3 week titration. Participants were excluded if they had any significant psychiatric comorbidity including $\mathrm{ADHD}, \mathrm{AN}$, or $\mathrm{BN}$. In treating patients who binge, abstinence is one of the first treatment targets. Binge abstinence in this study was described as 4-week bingeeating (BE) response cessation (100\%). At 11 weeks, the 4-week BE response cessation rate was significantly greater for all doses of LDX than placebo; notably, $50 \%$ of participants taking $70 \mathrm{mg}$ of LDX daily had $100 \%$ BE response over 4 weeks and $42 \%$ at $50 \mathrm{mg}$ LDX daily. According to a systematic review of the three clinical studies of LDX, the pooled number needed to treat (NNT) for remission (4-week binge eating cessation) was 4 (95\% CIs 4-6) and the number needed to harm (NNH) for discontinuation due to an adverse event for LDX was 44 (95\% CI 23-1971) compared to placebo. [56]. LDX-reduced binge-eating episodes (at doses of 50 or $70 \mathrm{mg}$ daily) with a good effect size (0.83-0.97) and 


\section{Conclusion}

participants also lost weight (approximately 5-6\% weight reduction).

Given suggested dopaminergic neurotransmitter dysfunction in overeating, stimulant medications that affect dopamine might help with binge eating and overeating. LDX is a prodrug of D-amphetamine, which blocks the reuptake of norepinephrine and dopamine. Long-term studies are ongoing and will be important to clarify if these promising, positive effects on binge eating are maintained. It will also be important to follow-up whether the positive effects from this 8 week study is maintained in the ongoing long-term LDX study, although LDX appears to be a promising treatment option for BED based on short-term data. Studies in children and adolescents are needed. This medication is the first FDA-approved drug to treat BED that might possibly be used to treat both ADHD and BED. The efficacy of this medication in both ADHD and short term to treat BED suggests a need to investigate possible common neurobehavioral targets (such as impulsivity or another factor).

Although atomoxetine is not approved by the FDA to treat binge eating, it is approved for the treatment of ADHD in adults and children [57]. A small study of atomoxetine, a selective norepinephrine reuptake inhibitor, was found to reduce binge eating in adults [58]. The central norepinephrine system may be implicated in eating behavior. While the prominent effect of atomoxetine is on norepinephrine reuptake, atomoxetine may also affect response inhibition and posterior attentional systems [59] and lead to improvements in inhibitory control. ATM could theoretically target impulsivity in both ADHD and BED through frontal and prefrontal brain circuitries. A case report of atomoxetine employed to treat [60] a youth with ADHD, obesity, and MC4R suggested a need for additional study. To date, no studies regarding the efficacy of atomoxetine in youth with BED have been performed.

There is a compelling need to study the cognitive and neurobiological mechanisms of BED, particularly in the context of ADHD, in order to develop more targeted treatments based on neurobehavioral underpinnings (such as impulsivity and inattention).

There is growing evidence of a relationship between ADHD and eating disorders, particularly binge-eating behaviors (such as seen in BED and BN). Future longitudinal studies are needed to elucidate these relationships using prospective studies. There is a need to explore the neurobehavioral underpinnings of these disorders (for example through neuroimaging studies) in order to develop targeted treatments for ADHD and BED.

\section{Acknowledgments}

Funding has been provided by the National Institutes of Health, National Institute of Mental Health, by Grant K23MH083000. 


\section{Compliance with ethics guidelines}

\section{Conflict of Interest}

Shauna P. Reinblatt has received royalty from the Osler Institute for Past Board Review CME Lectures; non-financial support (travel) from Sunovion, Actavis, and Alcobra; and consulting for the National Board of Medical Examiners.

\section{Human and animal rights and informed consent}

This article does not contain any studies with laboratory animals performed by any of the authors. With regard to the authors' research cited in this paper, all institutional and national guidelines for human studies were followed (IRB approved studies).

\section{References and Recommended Reading}

Papers of particular interest, published recently, have been highlighted as:

- Of importance.

$\bullet \quad$ Of major importance

1. Willcutt EG. The prevalence of DSM-IV attention-deficit/hyperactivity disorder: a meta-analytic review. Neurotherapeutics. 2012;9(3):490-9.

2. American Psychiatric Association. Diagnostic and statistical manual of mental health disorders: DSM-5 (5th ed). 5th ed. Washington DC: American Psychiatric Publishing; 2013.

3. Hudson JI, Hiripi E, Pope Jr HG, Kessler RC. The prevalence and correlates of eating disorders in the National Comorbidity Survey Replication. Biol Psychiatry. 2007;61(3):348-58.

4. Sobanski E. Psychiatric comorbidity in adults with attention-deficit/hyperactivity disorder (ADHD). Eur Arch Psychiatry Clin Neurosci. 2006;256 Suppl 1:i2631.

5. Mattos P, Saboya E, Ayrao V, Segenreich D, Duchesne $\mathrm{M}$, Coutinho G. Comorbid eating disorders in a Brazilian attention-deficit/hyperactivity disorder adult clinical sample. Rev Bras Psiquiatr. 2004;26(4):24850 .

6. Surman CB, Randall ET, Biederman J. Association between attention-deficit/hyperactivity disorder and bulimia nervosa: analysis of 4 case-control studies. J Clin Psychiatry. 2006;67(3):351-4.

7. Wentz E, Lacey JH, Waller G, Rastam M, Turk J, Gillberg C. Childhood onset neuropsychiatric disorders in adult eating disorder patients. A pilot study. Eur Child Adolesc Psychiatry. 2005;14(8):431-7.

8. Yates WR, Lund BC, Johnson C, Mitchell J, McKee P. Attention-deficit hyperactivity symptoms and disorder in eating disorder inpatients. Int J Eat Disord. 2009;42(4):375-8.

9. Mikami AY, Hinshaw SP, Patterson KA, Lee JC. Eating pathology among adolescent girls with attention- deficit/hyperactivity disorder. J Abnorm Psychol. 2008;117(1):225-35.

10. Blinder BJ, Cumella EJ, Sanathara VA. Psychiatric comorbidities of female inpatients with eating disorders. Psychosom Med. 2006;68(3):454-62.

11. Cortese S, Isnard P, Frelut ML, et al. Association between symptoms of attention-deficit/hyperactivity disorder and bulimic behaviors in a clinical sample of severely obese adolescents. Int J Obes (Lond). 2007;31(2):340-6.

12. Biederman J, Ball SW, Monuteaux MC, Surman CB, Johnson JL, Zeitlin S. Are girls with ADHD at risk for eating disorders? Results from a controlled, five-year prospective study. J Dev Behav Pediatr. 2007;28(4):302-7.

13. Mikami AY, Hinshaw SP, Arnold LE, et al. Bulimia nervosa symptoms in the multimodal treatment study of children with ADHD. Int J Eat Disord. 2010;43(3):248-59.

14.• Bleck JR, DeBate RD, Olivardia R. The comorbidity of ADHD and eating disorders in a nationally representative sample. J Behav Health Serv Res. 2014.

This article examines the comorbidity of ADHD and eating disorders (including clinical and subclinical behaviors) through secondary analysis of a large national sample.

15. Stulz N, Hepp U, Gachter C, Martin-Soelch C, Spindler A, Milos G. The severity of ADHD and eating disorder symptoms: a correlational study. BMC Psychiatry. 2013;13:44-244X-13-44.

16. Pagoto SL, Curtin C, Lemon SC, et al. Association between adult attention deficit/hyperactivity disorder and obesity in the US population. Obesity (Silver Spring). 2009;17(3):539-44. 
17. Waring ME, Lapane KL. Overweight in children and adolescents in relation to attention-deficit/hyperactivity disorder: results from a national sample. Pediatrics. 2008;122(1):e1-6.

18. Altfas JR. Prevalence of attention deficit/hyperactivity disorder among adults in obesity treatment. BMC Psychiatry. 2002;2:9.

19. Agranat-Meged AN, Deitcher C, Goldzweig G, Leibenson L, Stein M, Galili-Weisstub E. Childhood obesity and attention deficit/hyperactivity disorder: a newly described comorbidity in obese hospitalized children. Int J Eat Disord. 2005;37(4):357-9.

20. Holtkamp K, Konrad K, Muller B, et al. Overweight and obesity in children with attention-deficit/hyperactivity disorder. Int J Obes Relat Metab Disord. 2004;28(5):685-9.

21. Cortese S, Ramos Olazagasti MA, Klein RG, Castellanos FX, Proal E, Mannuzza S. Obesity in men with childhood ADHD: a 33-year controlled, prospective, followup study. Pediatrics. 2013;131(6):e1731-8.

22. Davis C, Levitan RD, Smith M, Tweed S, Curtis C. Associations among overeating, overweight, and attention deficit/hyperactivity disorder: a structural equation modelling approach. Eat Behav. 2006;7(3):266-74.

23. Seitz J, Kahraman-Lanzerath B, Legenbauer T, et al. The role of impulsivity, inattention and comorbid ADHD in patients with bulimia nervosa. PLoS One. 2013;8(5), e63891.

24. Cortese S, Bernardina BD, Mouren M. Attention-deficit/hyperactivity disorder (ADHD) and binge eating. Nutr Rev. 2007;65(9):404-11.

25. Matherne CE, Tanofsky-Kraff M, Altschul AM, et al. A preliminary examination of loss of control eating disorder (LOC-ED) in middle childhood. Eat Behav. 2015;18:57-61.

26. Reinblatt SP, Mahone EM, Tanofsky-Kraff M, et al. Pediatric loss of control eating syndrome: association with attention-deficit/hyperactivity disorder and impulsivity. Int J Eat Disord. 2015;48(6):580-8.

27. Bleck J, DeBate RD. Exploring the co-morbidity of attention-deficit/hyperactivity disorder with eating disorders and disordered eating behaviors in a nationally representative community-based sample. Eat Behav. 2013;14(3):390-3.

28. Steadman KM, Knouse LE. Is the relationship between ADHD symptoms and binge eating mediated by impulsivity? J Atten Disord. 2014.

29.•• Sonneville KR, Calzo JP, Horton NJ, et al. Childhood hyperactivity/inattention and eating disturbances predict binge eating in adolescence. Psychol Med. 2015:1-10.

This study examines risks factors (such as childhood ADHD) for developing adolescent binge eating in a large cohort study of children in the United Kingdom.

30. Reinblatt SP, Leoutsakos JM, Mahone EM, Forrester S, Wilcox HC, Riddle MA. Association between binge eating and attention-deficit/hyperactivity disorder in two pediatric community mental health clinics. Int J Eat Disord. 2015;48(5):505-11.

31. Ptacek R, Kuzelova H, Stefano GB, et al. Disruptive patterns of eating behaviors and associated lifestyles in males with ADHD. Med Sci Monit. 2014;20:608-13.

32. Bosanac P, Kurlender S, Stojanovska L, et al. Neuropsychological study of underweight and "weightrecovered" anorexia nervosa compared with bulimia nervosa and normal controls. Int J Eat Disord. 2007;40(7):613-21.

33. Werthmann J, Jansen A, Roefs A. Worry or craving? A selective review of evidence for food-related attention biases in obese individuals, eating-disorder patients, restrained eaters and healthy samples. Proc Nutr Soc. 2015;74(2):99-114.

This review examines the influence of attention bias on eating behaviors.

34. Higgs S. Manipulations of attention during eating and their effects on later snack intake. Appetite. 2015;92:287-94.

35. Wodka EL, Mahone EM, Blankner JG, et al. Evidence that response inhibition is a primary deficit in ADHD. J Clin Exp Neuropsychol. 2007;29(4):345-56.

36. Volkow ND, Wang GJ, Fowler JS, et al. "Nonhedonic" food motivation in humans involves dopamine in the dorsal striatum and methylphenidate amplifies this effect. Synapse. 2002;44(3):175-80.

37. Silvetti M, Wiersema JR, Sonuga-Barke E, Verguts T. Deficient reinforcement learning in medial frontal cortex as a model of dopamine-related motivational deficits in ADHD. Neural Netw. 2013;46:199-209.

38. Gearhardt AN, Boswell RG, White MA. The association of "food addiction" with disordered eating and body mass index. Eat Behav. 2014;15(3):427-33.

39. Davis C. The epidemiology and genetics of binge eating disorder (BED). CNS Spectr. 2015:1-8.

This manuscript features a thorough, recent review of the genetics of binge eating disorder.

40. Jonsson EG, Nothen MM, Grunhage F, et al. Polymorphisms in the dopamine D2 receptor gene and their relationships to striatal dopamine receptor density of healthy volunteers. Mol Psychiatry. 1999;4(3):290-6.

41. Nederkoorn C, Smulders FT, Havermans RC, Roefs A, Jansen A. Impulsivity in obese women. Appetite. 2006;47(2):253-6.

42. Kelly NR, Bulik CM, Mazzeo SE. Executive functioning and behavioral impulsivity of young women who binge eat. Int J Eat Disord. 2013;46(2):127-39.

43. Galioto R, Spitznagel MB, Strain G, et al. Cognitive function in morbidly obese individuals with and without binge eating disorder. Compr Psychiatry. 2012;53(5):490-5.

44. Hartmann AS, Rief W, Hilbert A. Laboratory snack food intake, negative mood, and impulsivity in youth with ADHD symptoms and episodes of loss of control eating. Where is the missing link? Appetite. 2012;58(2):672-8.

45. Karhunen LJ, Vanninen EJ, Kuikka JT, Lappalainen RI, Tiihonen J, Uusitupa MIJ. Regional cerebral blood flow 
during exposure to food in obese binge eating women. Psychiatry Res - Neuroimaging. 2000;99(1):29-42.

46.• Wang G, Geliebter A, Volkow ND, et al. Enhanced striatal dopamine release during food stimulation in binge eating disorder. Obesity. 2011;19(8):1601-8.

This study was the first PET study to examine dopamine changes in the brain in BED with a food stimulus.

47. Castellanos FX, Giedd JN, Eckburg P, et al. Quantitative morphology of the caudate nucleus in attention deficit hyperactivity disorder. Am J Psychiatry. 1994;151(12):1791-6.

48. Balodis IM, Grilo CM, Kober H, et al. A pilot study linking reduced fronto-striatal recruitment during reward processing to persistent bingeing following treatment for binge-eating disorder. Int J Eat Disord. 2014;47(4):376-84.

49. Geliebter A, Benson L, Pantazatos SP, Hirsch J, Carnell $\mathrm{S}$. Greater anterior cingulate activation and connectivity in response to visual and auditory high-calorie food cues in binge eating: preliminary findings. Appetite. 2015.

50. Schienle A, Schafer A, Hermann A, Vaitl D. Binge-eating disorder: reward sensitivity and brain activation to images of food. Biol Psychiatry. 2009;65(8):654-61.

51. Lock J, Garrett A, Beenhakker J, Reiss AL. Aberrant brain activation during a response inhibition task in adolescent eating disorder subtypes. Am J Psychiatry. 2011;168(1):55-64.

52. Bledsoe JC, Semrud-Clikeman M, Pliszka SR. Anterior cingulate cortex and symptom severity in attentiondeficit/hyperactivity disorder. J Abnorm Psychol. 2013;122(2):558-65.

53. Marsh R, Steinglass JE, Gerber AJ, et al. Deficient activity in the neural systems that mediate self-regulatory control in bulimia nervosa. Arch Gen Psychiatry. 2009;66(1):51-63.
54. Marsh R, Horga G, Wang Z, et al. An FMRI study of selfregulatory control and conflict resolution in adolescents with bulimia nervosa. Am J Psychiatry. 2011;168(11):1210-20.

55.• McElroy SL, Hudson JI, Mitchell JE, et al. Efficacy and safety of lisdexamfetamine for treatment of adults with moderate to severe binge-eating disorder: a randomized clinical trial. JAMA Psychiatry. 2015;72(3):23546.

This manuscript describes a randomized controlled trial of lisdexamfetamine to reduce binge eating (at doses of $50 \mathrm{mg}$ and $70 \mathrm{mg} /$ day) in adults.

56. Citrome L. Lisdexamfetamine for binge eating disorder in adults: a systematic review of the efficacy and safety profile for this newly approved indication-what is the number needed to treat, number needed to harm and likelihood to be helped or harmed? Int J Clin Pract. 2015;69(4):410-21.

57. Vaughan B, Fegert J, Kratochvil CJ. Update on atomoxetine in the treatment of attention-deficit/hyperactivity disorder. Expert Opin Pharmacother. 2009;10(4):669-76.

58. McElroy SL, Guerdjikova A, Kotwal R, et al. Atomoxetine in the treatment of binge-eating disorder: a randomized placebo-controlled trial. J Clin Psychiatry. 2007;68(3):390-8.

59. Bymaster FP, Katner JS, Nelson DL, et al. Atomoxetine increases extracellular levels of norepinephrine and dopamine in prefrontal cortex of rat: a potential mechanism for efficacy in attention deficit/ hyperactivity disorder. Neuropsychopharmacology. 2002;27(5):699-711.

60. Pott W, Albayrak O, Hinney A, Hebebrand J, Pauli-Pott U. Successful treatment with atomoxetine of an adolescent boy with attention deficit/hyperactivity disorder, extreme obesity, and reduced melanocortin 4 receptor function. Obes Facts. 2013;6(1):109-15. 